\title{
Thermal diffusivity of seasonal snow determined from temperature profiles
}

\author{
H.J. Oldroyd ${ }^{\mathrm{a}, *}$, C.W. Higgins ${ }^{\mathrm{a}, \mathrm{b}}$, H. Huwald ${ }^{\mathrm{a}}$, J.S. Selker ${ }^{\mathrm{b}}$, M.B. Parlange ${ }^{\mathrm{a}}$ \\ ${ }^{a}$ School of Architecture, Civil and Environmental Engineering, École Polytechnique Fédérale de Lausanne, Station 2, 1015 Lausanne, Switzerland \\ ${ }^{\mathrm{b}}$ Department of Biological and Ecological Engineering, Oregon State University, Corvallis, OR, USA
}

\section{A R T I C L E I N F O}

Article history:

Available online $\mathrm{xxxx}$

\section{Keywords:}

Heat diffusion

Porous media

Snow temperature measurements

Thermal conductivity

Thermal diffusivity

\begin{abstract}
A B S T R A C T
Thermal diffusivity of snow is an important thermodynamic property associated with key hydrological phenomena such as snow melt and heat and water vapor exchange with the atmosphere. Direct determination of snow thermal diffusivity requires coupled point measurements of thermal conductivity and density, which continually change due to snow metamorphism. Traditional methods for determining these two quantities are generally limited by temporal resolution. In this study we present a method to determine the thermal diffusivity of snow with high temporal resolution using snow temperature profile measurements. High resolution (between 2.5 and $10 \mathrm{~cm}$ at $1 \mathrm{~min}$ ) temperature measurements from the seasonal snow pack at the Plaine-Morte glacier in Switzerland are used as initial conditions and Neumann (heat flux) boundary conditions to numerically solve the one-dimensional heat equation and iteratively optimize for thermal diffusivity. The implementation of Neumann boundary conditions and a ttest, ensuring statistical significance between solutions of varied thermal diffusivity, are important to help constrain thermal diffusivity such that spurious high and low values as seen with Dirichlet (temperature) boundary conditions are reduced. The results show that time resolved thermal diffusivity can be determined from temperature measurements of seasonal snow and support density-based empirical parameterizations for thermal conductivity.
\end{abstract}

() 2012 Elsevier Ltd. All rights reserved.

\section{Introduction}

Snow thermal diffusivity is an important thermodynamic property in snow hydrology because it regulates heat diffusion in the snow as defined by the heat equation [1]. Hence, it is an important parameter for understanding heat transfer and predicting snowmelt [2], and is therefore applicable to water resources management activities such as agriculture, hydropower and municipal water usages [3]. Furthermore, thermal diffusivity can be useful in improved prediction of snowmelt timing and streamflow discharge because typical snowmelt models are tuned to air temperature, and therefore, lack a full physical basis [4]. Heat transfer in snow occurs by conduction through the ice matrix, by conduction through air-filled pore spaces, by latent heat exchanges of water vapor (condensation and sublimation) [3] and by radiative heating $[5,3,6]$. In addition, convection [7-9] and wind pumping [10,11] can also play a role. However, these mechanisms are less well understood. Better temporal resolution in heat transfer studies may allow for improved understanding of these mechanisms because they occur at relatively short time scales.

Snow is a porous medium whose physical properties evolve in time, and therefore can be difficult to characterize. It continuously

\footnotetext{
* Corresponding author.

E-mail address: holly.oldroyd@epfl.ch (H.J. Oldroyd).
}

undergoes morphological changes in grain size and shape, density, and bonding due to metamorphism, all of which affect the snow thermal properties [3]. Thermal conductivity of snow is also anisotropic, having different values in horizontal and vertical directions [12]. A better understanding of heat transfer processes in snow and their natural impacts requires a better understanding of how thermophysical properties vary in time [13]. Due to the labor intensity of some measurement techniques, such as the excavation of snow pits, the potential temporal and spatial resolution of snow property measurements is limited. Furthermore, some measurement techniques might actually alter the snow properties being measured by disrupting its structure [14].

Direct determination of thermal diffusivity requires measurements (either in situ or in extracted samples) of two quantities: thermal conductivity and snow density. Generally, density measurements require snow pit excavation and gravimetric analysis of individual samples from varying depths. Therefore, snow density measurements with high temporal resolution are difficult to obtain. For thermal conductivity, several techniques exist which vary greatly in cost, ease of implementation, accuracy and spatial and temporal resolution. Some of these methods are described in Section 2, and a more rigorous summary and critique of several of the techniques was presented by Sturm et al. [15]. Many of these methods require specialized, relatively expensive equipment in addition to requiring substantial effort to obtain measurements at high spatial and temporal resolution. 
An alternative approach is to measure snow temperatures and invert the heat equation to obtain the thermal conductivity or thermal diffusivity of snow. The major advantages of this approach are that temperature is relatively inexpensive to measure and can provide many more options for spatial and temporal resolution. As an example of the inversion approach, Brandt and Warren [16] successfully used a one-dimensional vertical finite difference scheme with Dirichlet boundary conditions (specifying temperature in time) and a stationary density profile to optimize for thermal conductivity of snow at the South Pole. However, the Brandt and Warren [16] study is not representative of ephemeral, seasonal snow packs of hydrological importance because the diurnal solar forcing was atypical (polar night for 6 months) and snow temperatures were extremely low $\left(\leqslant-40^{\circ} \mathrm{C}\right)$. In relatively warm snowpacks latent heat transfer can account for a significant portion of the overall heat transfer [15]. Due to these complications, it was unclear that the method used by Brandt and Warren [16] could be successful for relatively warm snowpacks with the strong diurnal forcing experienced by seasonal snow.

For the present study the inversion approach of Brandt and Warren [16] was extended to determine thermal diffusivity for seasonal snow with the addition of two key steps. First, Neumann boundary conditions (specifying heat flux in time, as determined by the temperature measurements) must be used for the simulation so the predicted temperatures are not artificially constrained, and second, a statistical test is integrated into the analysis to determine when there is sufficient information within the temperature profiles to converge to a unique solution. In this paper this new approach was used to find the thermal diffusivity for seasonal snow on the Plaine-Morte glacier, an important water reservoir in the Bernese Alps of Switzerland. High resolution (between 2.5 and $10 \mathrm{~cm}$ at $1 \mathrm{~min}$ ) temperature measurements were used as initial and boundary conditions to numerically solve the one-dimensional heat equation, and iteratively optimize for thermal diffusivity. The temperature measurement probe used in this study is relatively inexpensive, easy to install and allows for monitoring throughout a season. Section 2 of this paper discusses and compares some of the existing techniques to obtain the thermal diffusivity of snow. In Section 3, the experiment site and temperature measurement techniques are described. The proposed method to determine time-resolved thermal diffusivity of seasonal snow is presented in Section 4. Finally, the results and error analyses are presented and discussed along with recommendations for subsequent uses of the new measurement probe and methods for determining thermal diffusivity.

\section{Thermal diffusivity and measurement techniques}

Thermal diffusivity is a combination of the thermal properties of a medium which govern the heat diffusion rate through that medium according to the heat equation

$\frac{\partial T}{\partial t}=\alpha \frac{\partial^{2} T}{\partial z^{2}}$

where $\alpha$ is the thermal diffusivity, $T$ is temperature, $z$ is distance (in our case vertical depth) and $t$ is time [1]. The thermal diffusivity is defined as

$\alpha=\frac{\kappa}{\rho C_{p}}$,

where $\kappa$ is the thermal conductivity, $\rho$ is the density and $C_{p}$ is the specific heat capacity. Since snow is a porous media comprised of ice matrices and air-filled pores, its thermal properties are generally described as being effective values at averaged macro scales [17,3]. Throughout this paper thermal properties are to be considered their effective values in the vertical component, considering snow's anisotropy. Heat conduction through the solid ice matrix is the dominant heat transfer mechanism because the thermal conductivity of ice is approximately two orders of magnitude greater than that of air [3]. Accordingly, snow microstructure, grain size, shape and intergranular bonding, strongly influence the thermal conductivity [13]. In addition, latent heat transfer can also play a significant role in relatively warm snow [3]. However, latent heat transfer is also a diffusive mechanism which occurs along a temperature gradient, so its effects can be naturally lumped into an effective thermal diffusivity $[18,15,17,3]$.

Several techniques exist for measuring thermal conductivity. One such method is to use a heated plate to study the steady-state heat flow across an extracted block of snow [19]. Once at equilibrium, the temperature gradient across the sample block is used to calculate the thermal conductivity. Since the snow sample is heated from one end, care must be taken to insulate the edges such that the heat transfer occurs only across the sample [19,15]. In addition, the imposed temperature gradient may cause the snow sample to undergo metamorphism [15]. Another highly technical approach for determining thermal conductivity is based on microstructure tomography (e.g. $[20,12])$. This methodology uses tomographic 3D images of snow microstructure and the steady-state heat transport equation to determine the effective thermal conductivity by separating the heat transfer over the ice matrix and pore spaces for a volumetric snow sample [12]. Beyond the specialized equipment necessary for these types of analyses, the methods are relatively labor intensive and require careful extraction and storage of snow samples. Hence, obtaining measurements with high spatial and temporal resolution with this approach is impractical.

Another approach for measuring thermal conductivity uses the transient heat transfer resulting from an unsteady heat input (and often the subsequent cooling) [21,22]. Sturm et al. [15] describe some of the various instrument configurations that have been used with the transient heat transfer approach, and mention that needle probe or point heat source configurations are ideal because they are less likely to disturb the snow. Generally, the needle probe consists of a thin needle of which one end is heated and the other end monitors the temperature change between the two sections. In concept, the analytical solution for heat diffusion in an infinite wire predicts that the temperature change over the needle is linearly related to the natural log of time, and the thermal conductivity is proportional to the slope of this line [23]. These heat probes have been used in cold chambers [24,25], inserted into walls of snow pits $[24,22,15]$, and naturally covered by snow fall [23]. The transient heat probe method for determining thermal conductivity provides more flexibility in terms of spatial and temporal resolution than the steady-state method. The needle probes, though small, may still significantly alter the snow structure [14], and Calonne et al. [12] show that thermal conductivities determined by the needle probe methods are systematically lower than those determined by other methods.

Empirical parameterizations, which relate the thermal conductivity to the snow density, have also been proposed. Sturm et al. [15] presented a parameterization based on a comprehensive compilation of snow density and thermal conductivity measurements from several techniques and studies. Other empirical, densitybased parameterizations have also been proposed by Calonne et al. [12] (determined from tomographical, heated plate, and the needle probe techniques), Aggarwal et al. [26] (determined by the needle probe technique), and Yen [27] (determined from a compilation of unnumbered investigations). All of these parameterizations have a wide range of scatter, usually attributed to the differences in snow microstructure and bonding that can occur for a given snow density. Furthermore, these parameterizations require knowledge of the snow densities which, as mentioned 
previously, are dynamic and difficult to monitor with high temporal resolution. Often these types of parameterizations are used in various heat and mass transfer models for snow. Some examples are a catchment-scale snowmelt model [2], a glacier mass balance model [28], a climatological atmospheric warming study using englacial temperatures [29], in a near-surface model to be used by backcountry avalanche forecasters [30], and for comparison with a micro-structure based snow cover model [31].

Other methods that use snow temperature profiles to determine thermal diffusivity include spectral or analytical techniques. The spectral method tracks the amplitude ratio and phase shift of the diurnal temperature signal as it propagates into the snowpack [32]. This method is ideally applied over several diurnal cycles in a stationary medium and hence, is not well suited to snow because it cannot account for the transient features of the snowpack. Another inversion approach might be to use the existing analytical solutions to the initial boundary value problem that can be found in chapter 3 of Carlslaw and Jaeger [1]. This was the initial approach used in the present study, but it was found that the analytical solution (not shown herein) was more computationally expensive to evaluate and numerically unstable (Gibb's ringing near the boundaries) than numerical solutions to the heat equation.

The method proposed here to determine thermal diffusivity of seasonal snow with high temporal resolution is a numerical inversion of the heat equation. As mentioned previously, Brandt and Warren [16] successfully used a one-dimensional vertical finite difference scheme with Dirichlet boundary conditions to optimize for effective thermal conductivity from temperature measurements obtained at the South Pole. They used a density profile determined from a linear fit of densities measured in two snow pits separated in time by nearly one year (January 1992 and December 1992). Brandt and Warren [16] calculate thermal conductivity on 15 min intervals, and report 9-day averages with error bars representing conductivities with $\pm 10 \%$ relative error.

Although Brandt and Warren [16] have shown that on average, this type of method can successfully determine thermal conductivity for relatively cold snowpacks with little diurnal forcing, it is unclear that the method would be successful for typical seasonal snow packs. Reusser and Zehe [33] used the same method as Brandt and Warren [16], and applied it to temperature measurements of seasonal snow in the eastern Ore Mountains near the Czech-German boarder. They used a constant snow density and assumed thermal diffusivity to be constant over periods of 1 day or 5 days. In this case, although the snow itself and the environmental conditions were seasonal, the application of the method was not representative of the morphological variations associated with seasonal snow. Furthermore, Reusser and Zehe [33] present only one result for thermal diffusivity, $\alpha=5 \times 10^{-7} \mathrm{~m}^{2} \mathrm{~s}^{-1}$ and reported that $71 \%$ of the computed thermal diffusivity values were above an upper limit of $\alpha=1 \times 10^{-6} \mathrm{~m}^{2} \mathrm{~s}^{-1}$, the approximate thermal diffusivity of ice. Zhang and Osterkamp [34] presented a method which addresses this seemingly common artifact of spuriously high thermal diffusivities resulting from inversion methods. They proposed a finite difference method that retains higher order terms and thus requires at least five vertically aligned temperature measurements. Using this method as well as the standard finite difference method (neglecting higher order terms) both with Dirichlet boundary conditions, they determined thermal diffusivity from synthetic temperature profiles for permafrost and the soil active layer. Their comparison of these two methods showed that retaining higher order terms eliminated the spurious values that arose from the standard finite difference method. However, they also concluded that if higher order terms are retained, the temperature measurements used in the inversion must be much more accurate (as high as two orders of magnitude more depending on the spatial and temporal discretization [34]). Hence, this method may be impractical for use with field measurements. The present study proposes a similar heat equation inversion method for determining the thermal diffusivity of seasonal snow. However, we propose a method that naturally constrains the model such the spurious values of unrealistically high thermal diffusivity (as seen by Reusser and Zehe [33]) are reduced through the use of Neumann (heat flux) boundary conditions.

\section{Experimental set-up}

A field experiment was performed at the Plaine-Morte glacier, located in the Bernese Alps of Switzerland $\left(46.3863^{\circ} \mathrm{N}, 7.5178^{\circ}\right.$ E, 2750 m elevation) from 29 January to 4 March 2008 . The glacier is approximately $2 \mathrm{~km}$ wide, $5 \mathrm{~km}$ long and essentially flat, providing a relatively horizontally homogeneous snow field and atmospheric fetch.

To obtain vertical profiles of temperature within the seasonal snow on the glacier, a $2 \mathrm{~m}$ long, white (to reduce radiative effects) Polyamide 6 (PA6) pole was equipped with type-T $\left( \pm 1^{\circ} \mathrm{C}\right)$ thermocouples at $25 \mathrm{~mm}$ spacing prior to deployment. Thin circular grooves were machined into the pole so that when mounted, the thermocouples were flush with the surface of the pole (see the detail photo in Fig. 1). The measurement junction of each thermocouple is more than $10 \mathrm{~cm}$ from the point at which the wire emerges from the sensor core to assure accurate representation of the snow temperature. This pole will be referred to as the TCT probe for the remainder of the paper. The TCT probe was connected to a data logger (Campbell Scientific CR10X) by means of a 25 channel, solidstate multiplexer (Campbell Scientific AM25T). Since the number of thermocouples sampled was limited by the number of multiplexer channels, sampled thermocouple spacing varied between $40 \mathrm{~cm}$ and $2.5 \mathrm{~cm}$, and selection was made with the intent of having higher resolution temperature measurements near the snow surface throughout the season (maximum spacing for those used in the analysis was $5 \mathrm{~cm}$ ). The positive horizontal grid lines in Figs. 1 and 2 show the locations of the sampled thermocouples. Initially, the TCT probe was inserted vertically with the bottom $1 \mathrm{~m}$ in the snow, leaving $1 \mathrm{~m}$ above to measure subsequent snow accumulation. To maximize contact between the TCT probe and the snow, the TCT probe was firmly inserted with one smooth motion. Thermocouple temperatures were sampled every $1 \mathrm{~min}$.

In addition, a meteorological measurement station was erected at the site. Among the instruments sampled each 1 min were air temperature and relative humidity (Rotronic MP100A), snow height (Campbell Scientific SR50, acoustic range finder) and snow surface temperature (Apogee Instruments IRTS-P, infrared thermocouple sensor). On six occasions during the experiment, snow pits were dug and density profiles were measured by weighing samples of a known volume $\left(100 \mathrm{~cm}^{3}\right)$ with a standard SLF-Davos snow density kit. The vertical extent of the snow volume sampler is $3 \mathrm{~cm}$ and the snow density was sampled every $5 \mathrm{~cm}$ in depth. Fig. 2 shows the density evolution for the upper snowpack as well as snow height relative to the TCT probe. For all analyses we imposed a coordinate system defined by the TCT probe position. Hence, the matter below the vertical zero is the lower portion of the snowpack that the TCT probe does not reach, but where density measurements were made during the first snow pit excavation.

The acoustic range finder was located a few meters from the TCT probe and hence, could have reported slightly different snow height than what existed at the TCT probe location. Therefore, the snow height was also determined by inspection of the snow temperature profiles in a manner similar to the methods of Reusser and Zehe [33]. Fig. 1 presents an example of a 24-h period of snow temperature profiles. Profiles are used to determine the level of the snow surface by visual inspections over a 12 or 24 -h cycle for 


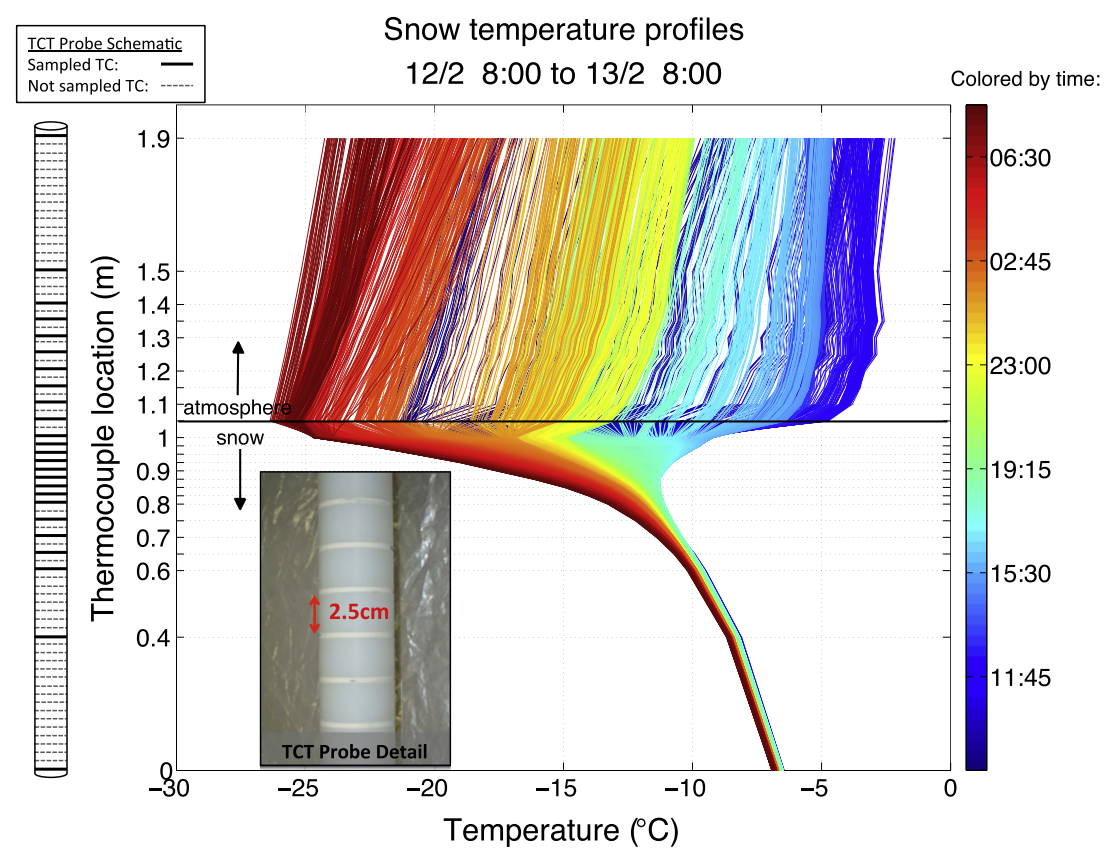

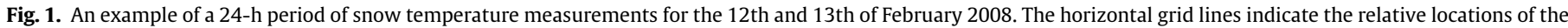
sampled thermocouples, which are also indicated in the TCT probe schematic. The inset figure shows a detail photo of the TCT probe.

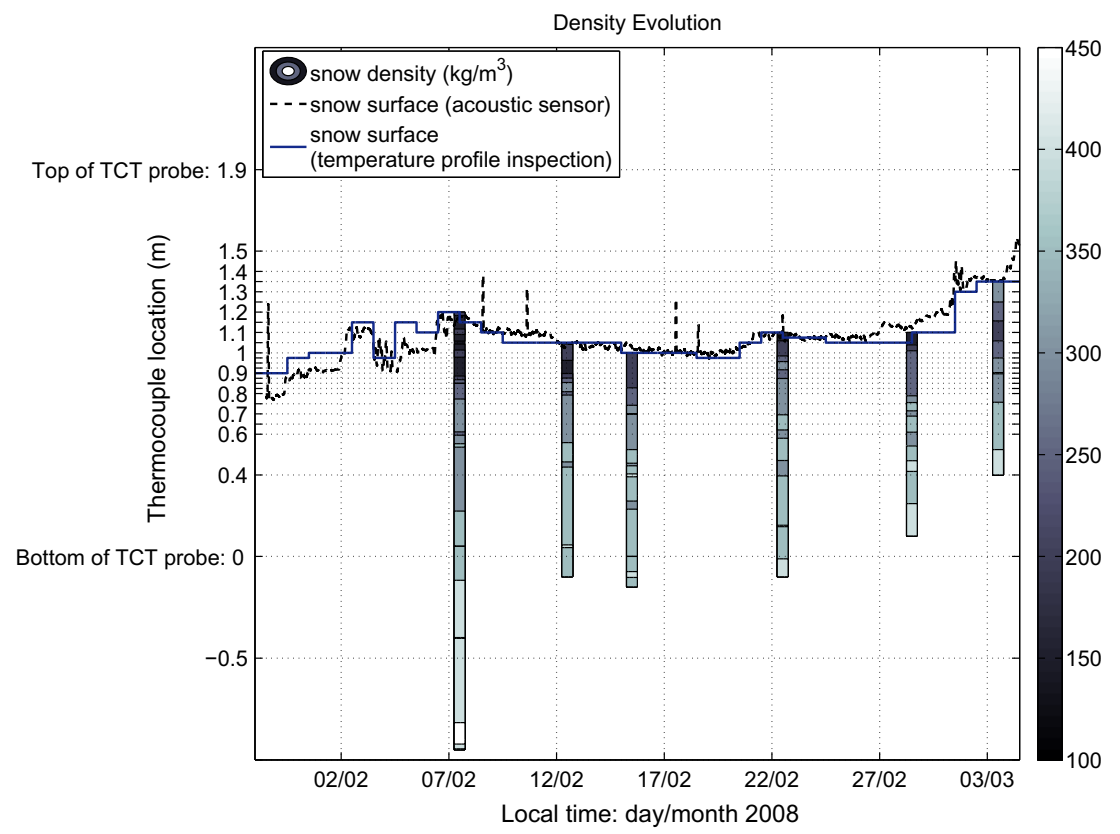

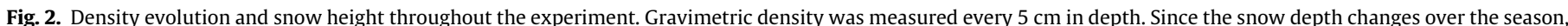

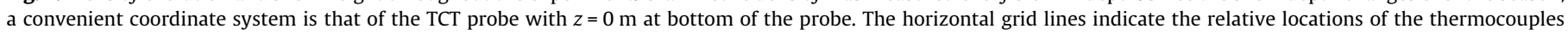
which were sampled throughout the experiment, with the exception of $z=-0.5 \mathrm{~m}$ that was included for spatial referencing of the first snow pit.

periods of no or minor precipitation. Snow has an insulating effect such that the temperature signals in the snow lag the thermal forcing at the surface and temperatures in the air are much more rapidly mixed. The surface is located near the sensor point where the ensemble of profiles over the considered period shows the most pronounced changes in gradient (kinks). Since this study required an approximate estimate of snow height (we only use thermocouples at least $10 \mathrm{~cm}$ below the snow surface), this snow height estimation method was sufficient.

\section{Methodology}

This study used the measured temperature profiles to determine thermal diffusivity of snow by inverting the heat equation (Eq. 1). More specifically, the heat equation was numerically solved in time and space given measured initial and boundary conditions for a range of thermal diffusivities. This was done iteratively, narrowing the range of effective thermal diffusivities until the RMS error between the measured and predicted temperatures 
was minimized. A flow chart describing the optimization routine is presented in Fig. 3.

The spatial domain, or specific thermocouple locations used for the modeled temperature predictions, was chosen with some competing considerations. This method determines a bulk thermal diffusivity over the domain simulated and so in principle, the extent of the domain should be minimized. However, having a larger number of temperature measurements in the domain allows for a better description of the physics (especially where large gradients and high degrees of curvature exist), and it provides more test points for comparing the respective RMS errors when optimizing for thermal diffusivity. The results presented herein were determined for a domain of seven internal measurement points between the top and bottom boundaries of the domain. On average the internal domain size was $25 \mathrm{~cm}$ and the changes in snow density over this depth range were small. The top boundary was chosen such that it is at least $10 \mathrm{~cm}$ below the snow surface to avoid regions where solar radiation may introduce non-linear effects to the heat transfer $[5,6]$.

The temporal domain used to simulate temperatures was $30 \mathrm{~min}$. Hence for each time step (every one minute) where thermal diffusivity is assigned, the RMS error is computed from the difference between the measured and predicted temperatures from $15 \mathrm{~min}$ into the past and future. Although the results are obtained for each one-minute time step, the temporal resolution of the results is $30 \mathrm{~min}$. Increasing the temporal domain naturally reduces the variation in the results; of course it also reduces the temporal resolution. In short, we solve for the bulk thermal diffusivity in the layer defined by the spatial domain as a function of time.

To test the robustness of the method, the search range for thermal diffusivity on the first iteration was wide and coarse, testing 30 values between $1 \times 10^{-9} \mathrm{~m}^{2} \mathrm{~s}^{-1}$ and $1 \times 10^{-1} \mathrm{~m}^{2} \mathrm{~s}^{-1}$. For each subsequent iteration, the search range was narrowed to test 20 values between 2 points on both sides of the value of thermal diffusivity with the lowest RMS error from the previous iteration. Iterations continued until the RMS error was changed by no more than $1 \times 10^{-4}$ and the value of effective thermal diffusivity that best represents the physical processes described by the heat equation was subsequently assigned. Fig. 3(c) contains an example of the final iteration for a single time step showing a clear, sharp RMS minimum associated with a particular thermal diffusivity.

The numerical predictions of temperature, or modeled temperatures, were determined by using MATLAB's partial differential equation (PDE) solver, pdepe, to solve the heat equation. This function solves a system of ordinary differential equations (ODEs), integrated in time, that result from the spatial discretization of the original PDE [35]. The initial condition was the first measured temperature profile in each 30 -min time segment, linearly interpolated to a profile with twice the original resolution (from 7 original measurement points to 14 points in the profile). Prior to calculation of the RMS error, simulated temperatures were interpolated back to the original spatial resolution of the measurements.

Neumann (heat flux) boundary conditions were used at the top and bottom of the domain. The Fourier heat fluxes at the boundaries were determined using finite differences of the temperature nodes on the internal boundaries and the adjacent external temperature nodes to calculate the respective temperature gradients, as shown in Fig. 4. All temperatures used are temperatures within the snowpack, not air or surface temperatures. Neumann boundary conditions are critical to this method because they increase the model's sensitivity to thermal diffusivity, constraining it such that spurious spikes of unrealistically high thermal diffusivity are reduced. This is achieved naturally since the simulated temperature profiles are not artificially constrained at the endpoints as is in the case of Dirichlet type boundary conditions. Since the solution is unconstrained, the trivial, steady-state solution of the heat

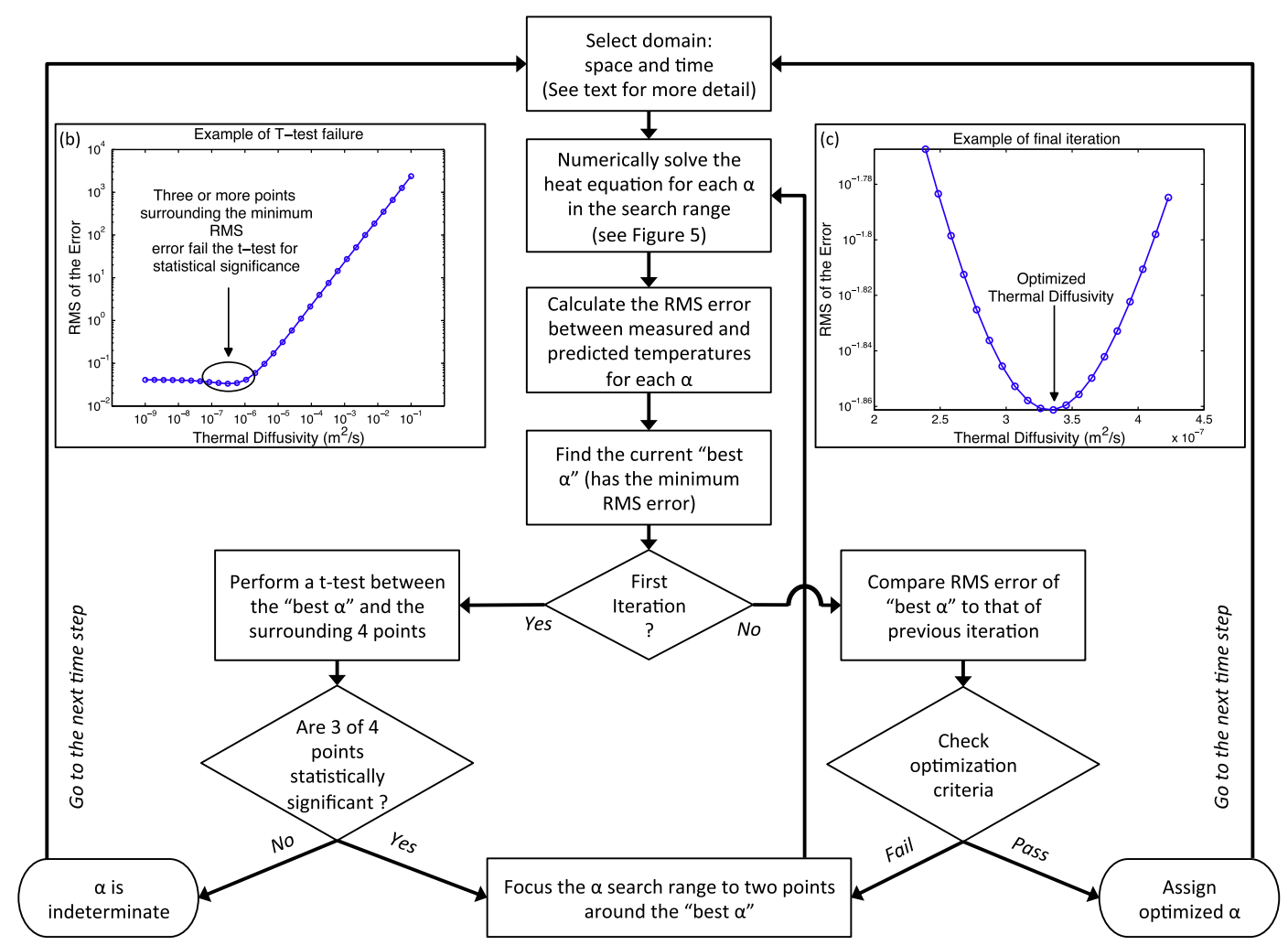

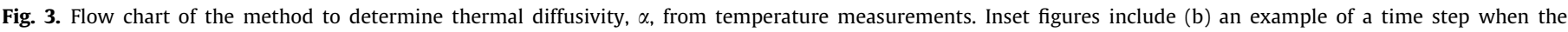
optimization fails the t-test, and (c) a time step with successful optimization. 


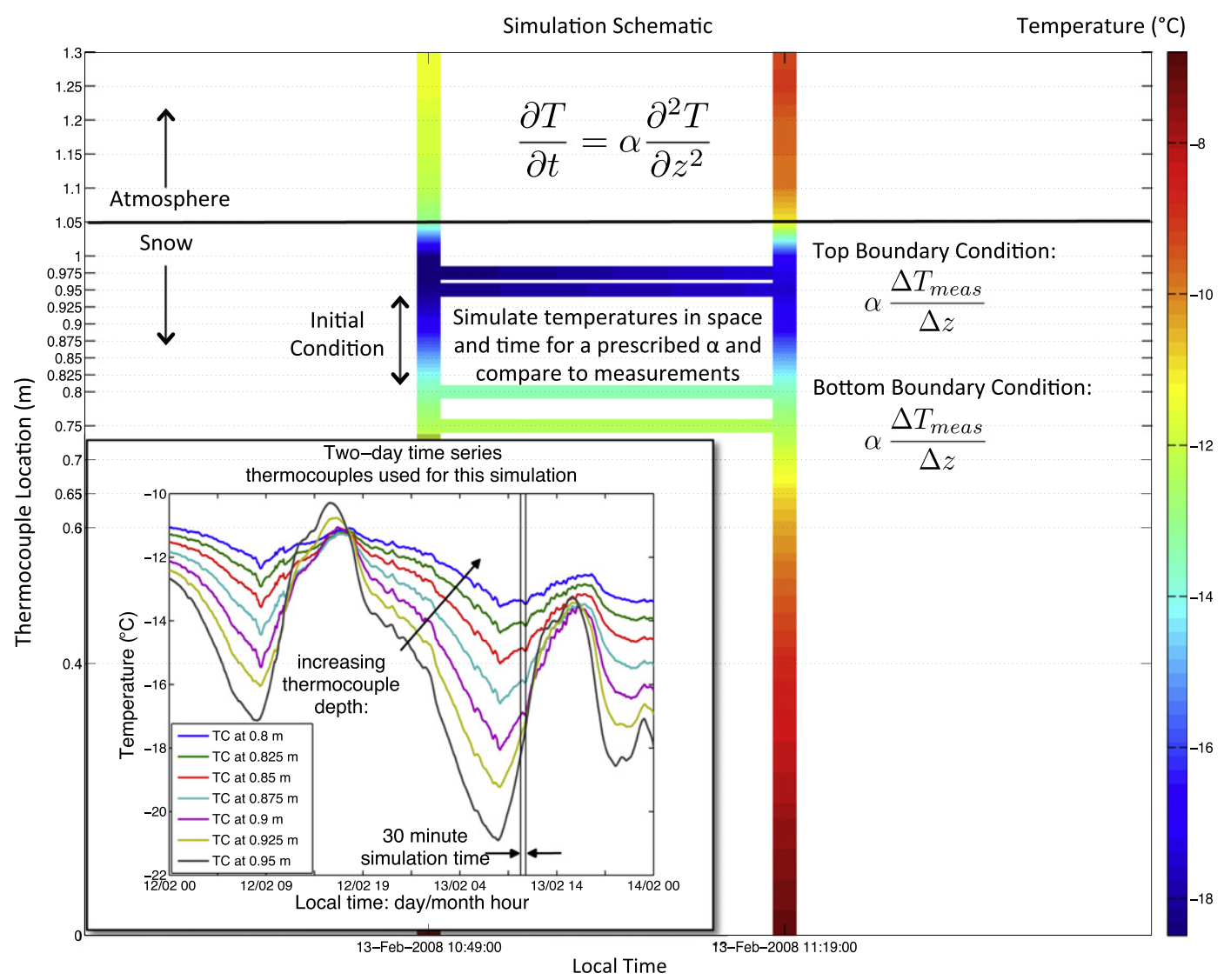

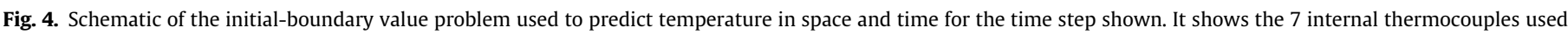

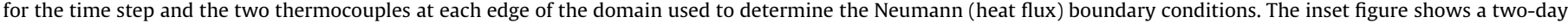
time series of the 7 internal thermocouples used for this period.

equation is excluded and consequently, the spuriously high values of diffusivity reported in other studies are avoided. The use of Neumann boundary conditions requires at least three measurements in the temperature profiles.

A further constraint on the model was applied for each time step by implementing a t-test on the initial search range for effective thermal diffusivity (the first optimization iteration). The t-test ensures that the RMS error associated with the minimum is statistically different from at least three of the four surrounding points (for example, when plotting RMS error versus thermal diffusivity). This step tends to eliminate spurious points of unrealistically low diffusivity by eliminating scenarios when optimization is attempted over a "flat" searching range, where no statistically clear minimum exists. Fig. 3(b) contains an example of a time step when the t-test indicated statistical insignificance, and thermal diffusivity was indeterminate.

\section{Results and discussion}

Time-resolved thermal diffusivities of seasonal snow determined from methods described in Section 4 are presented in Fig. 5 along with a line indicating the overall median value $\left(\alpha=2.5 \times 10^{-7}\right)$ of thermal diffusivity. The overall mean value of thermal diffusivity $\left(\alpha=3.9 \times 10^{-7}\right)$ is slightly higher. The results are shown with the density-based, empirical parameterizations for thermal conductivity [ $W \mathrm{~m}^{-1} \mathrm{~K}^{-1}$ ] of Sturm et al. [15],

$\kappa_{\text {eff }_{\text {stu }}}=0.138-1.01 \rho+3.233 \rho^{2} \quad\left\{0.156 \mathrm{~g} \mathrm{~cm}^{-3} \leqslant \rho \leqslant 0.6 \mathrm{~g} \mathrm{~cm}^{-3}\right\}$

$\kappa_{\text {eff }}=0.023+0.234 \rho \quad\left\{\rho<0.156 \mathrm{~g} \mathrm{~cm}^{-3}\right\}$,
Calonne et al. [12],

$\kappa_{\text {eff }_{\text {Cal }}}=2.5 \times 10^{-6} \rho^{2}-1.23 \times 10^{-4} \rho+0.024 \quad\left\{\rho\left[\mathrm{kg} \mathrm{m}^{-3}\right]\right\}$,

Aggarwal et al. [26]

$$
\begin{aligned}
\kappa_{\mathrm{eff}_{\mathrm{Agg}}}= & 0.00395+0.00084 \rho-1.7756 \times 10^{-6} \rho^{2} \\
& +3.80635 \times 10^{-9} \rho^{3} \quad\left\{\rho\left[\mathrm{kg} \mathrm{m}^{-3}\right]\right\},
\end{aligned}
$$

and Yen [27]

$\kappa_{\mathrm{eff}_{\text {Yen }}}=2.22362 \rho^{1.885} \quad\left\{\rho\left[\mathrm{Mg} \mathrm{m}^{-3}\right]\right\}$.

The densities that were measured during the six snow pit excavations and the specific heat of ice, $C_{p}=2090 \mathrm{~J} \mathrm{~kg}^{-1} \mathrm{~K}^{-1}$, were used to calculate the parameterized thermal diffusivities according to the above equations for thermal conductivity. In addition, indicators of the approximate 95\% confidence interval from Sturm et al. [15] density-based parameterization are also shown.

There is a high degree of variability in the thermal diffusivity time series. Despite the variability, the majority of the results are comparable to the density-based empirical parameterizations, and are within the bounds of the 95\% confidence interval reported in Sturm et al. [15] parameterizations. Furthermore, only 3.8\% of the thermal diffusivities are above $\alpha=1 \times 10^{-6} \mathrm{~m}^{2} \mathrm{~s}^{-1}$, the approximate thermal diffusivity of ice used as a theoretical upper limit by Reusser and Zehe [33]. Recall Reusser and Zehe [33] used a similar inversion technique, but with Dirichlet boundary conditions, and reported $71 \%$ of their thermal diffusivities above this limit. To quantify the improvement that imposing Neumann boundary conditions brought to the results, we also performed an inversion imposing Dirichlet boundary conditions. In the 


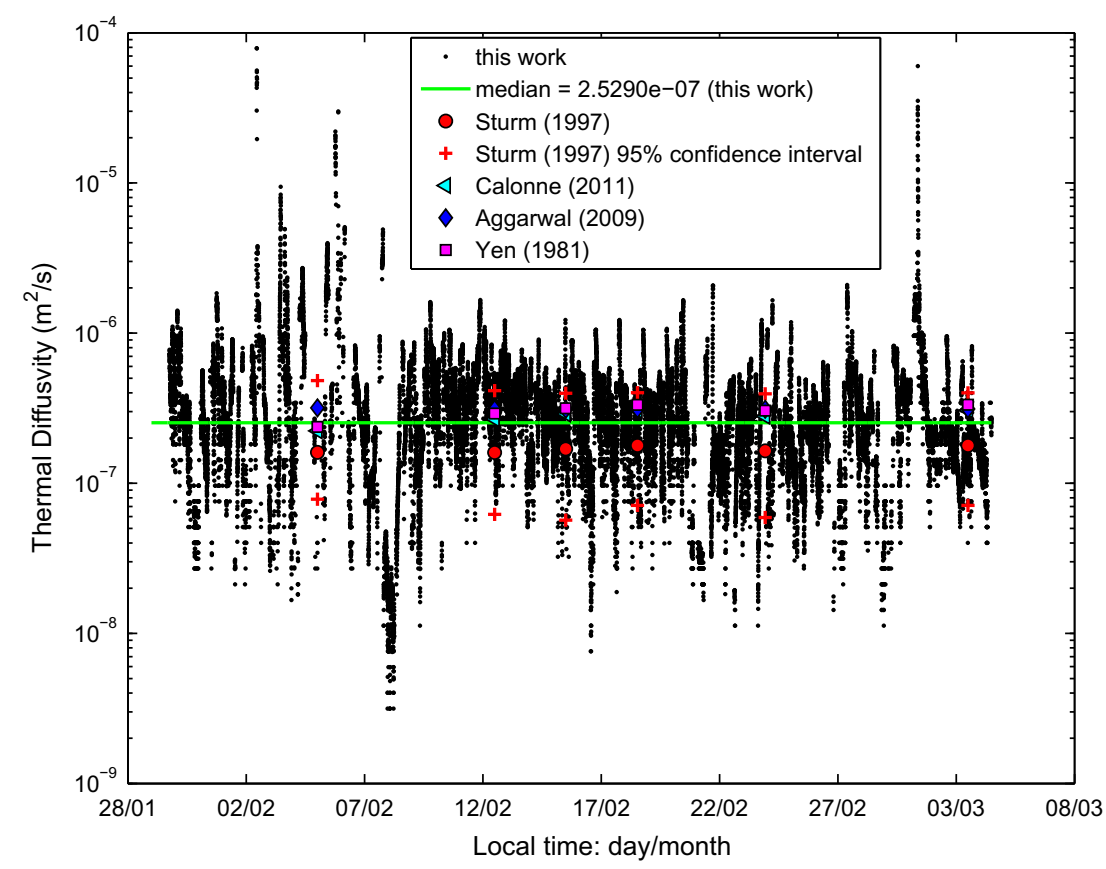

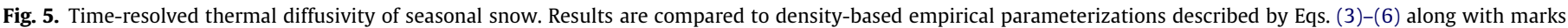
indicating the $95 \%$ confidence interval from Sturm et al. [15] parameterization.

Dirichlet case for the same temperature measurements, $17 \%$ were above $\alpha=1 \times 10^{-6} \mathrm{~m}^{2} \mathrm{~s}^{-1}$, and the diffusivity values were spread over a range of 10 orders of magnitude. Hence using Neumann boundary conditions provides a significant improvement to numerical inversion techniques for determining thermal diffusivity from temperature measurements with no extra computational expense.

The extreme values of thermal diffusivity in Fig. 5 and the rapid rate of change toward these extremities are not likely representative of physical changes in the thermal properties of the snow. To better understand this variability, additional analyzes were undertaken for a segment of the temperature data between 22 and 28 February 2008. The first such analysis explores the influence of the spatial domain to determine if it was too large to be characterized by a single value of thermal diffusivity for each time step. To address question of domain size, we use the same methodology to determine thermal diffusivity, but in a tri-layered scheme by dividing the original domain into three smaller domains of three temperature measurements each: top, middle and bottom. Recall that the thermal diffusivities reported in Fig. 5 were determined using an internal domain of 7 consecutive thermocouples (starting from $10 \mathrm{~cm}$ below the surface and increasing in depth). Fig. 6 show how the thermal diffusivities from the tri-layered scheme compare to those determined over the larger domain. The correlation plots (Figs. 6) show that the temperature signal in the top portion of the domain strongly influences the thermal diffusivity determined over the full domain because temperatures closer to the surface experience more change in time and exhibit higher gradients. All subdomains also show high degrees of variability and hence, the size of the spatial domain had little influence on the variations in the results. The tri-layer analysis also shows a trend of increasing thermal diffusivity with snow depth, which may be expected since thermal conductivity generally increases with snow density and density generally increases with snow depth.

The second analysis explores the possibility that uncertainty in the temperature measurements (type-T thermocouples: $\pm 1^{\circ} \mathrm{C}$ ) contributes to the high variability of the determined thermal diffusivities. Since this method depends on the relative accuracy between temperature measurements (e.g. $\partial T / \partial t$ and $\partial^{2} T / \partial z^{2}$ in the heat equation), it makes sense to assess the effects of noise in the temperature measurements. This was done using the same method and domain used to obtain the results in Fig. 5, but by adding three different levels of Gaussian noise to the temperature measurements, indicated by the standard deviation of the noise, $\sigma: \sigma=0.25^{\circ} \mathrm{C}\left(\Delta T_{\text {error,max }} \approx 1{ }^{\circ} \mathrm{C}\right), \sigma=0.33^{\circ} \mathrm{C}$ and $\sigma=0.70{ }^{\circ} \mathrm{C}$. Fig. 7 shows that noise in the temperature signal has little change in the degree of variability of the results. However, this analysis shows that noise in the temperature signal tends to cause an increase in the resulting thermal diffusivity. This is likely due to the need for increased diffusion to smooth out the noise in the temperature signal.

The third analysis explores the method's sensitivity to spatial discretization (or spatial resolution of temperature measurements) by using the same spatial domain size, but solving with half of the thermocouples used in the original results. Fig. 8 shows that decreasing the spatial discretization of the temperature measurements greatly increases the estimated thermal diffusivity, by almost two orders of magnitude in this case. Under-resolving the temperature profile leads to the underestimation of the curvature in the temperature profiles. Since the curvature is described mathematically by the $\partial^{2} T / \partial z^{2}$ term in the heat Eq. (1), the thermal diffusivity must increase to balance the equation. Decreasing the spatial resolution also increases the chances of excluding the peak in the temperature profile (refer to Fig. 1). This analysis also shows that decreasing the spatial resolution also tends to slightly increase the range of variability of the results, but not to the degree to explain the variability in Fig. 5.

The final study explores the temporal domain used to generate the modeled temperatures. First, spectral analysis was performed on the time series of thermal diffusivity presented in Fig. 5. It showed timescales smaller than 30 min exhibit characteristics that are typically attributed to noise, thus larger time domains should tend to smooth variations. Sharp spectral filtering at the $30 \mathrm{~min}$ time scale made almost no difference in the thermal diffusivity time series (data not shown). We also performed test cases of 2 , 3, 6 and 12-h temporal domains for which the variations in the 
(a)

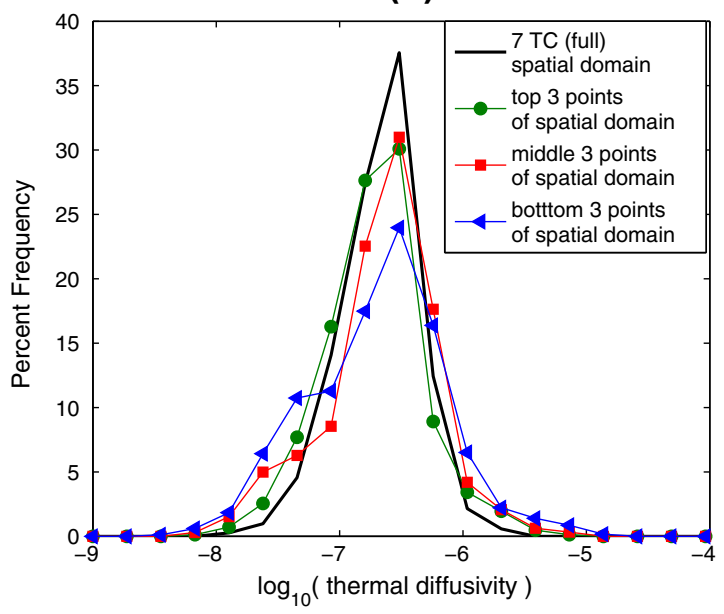

(c)

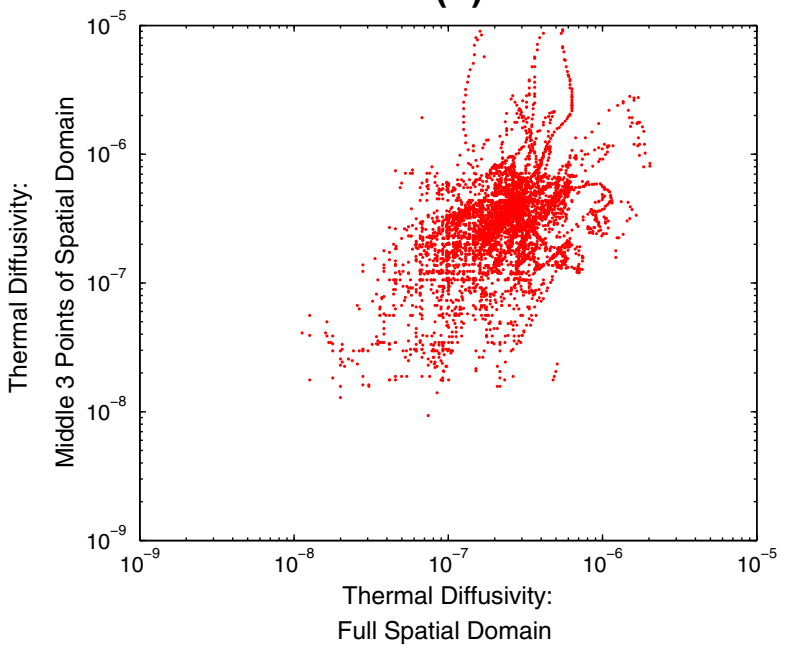

(b)

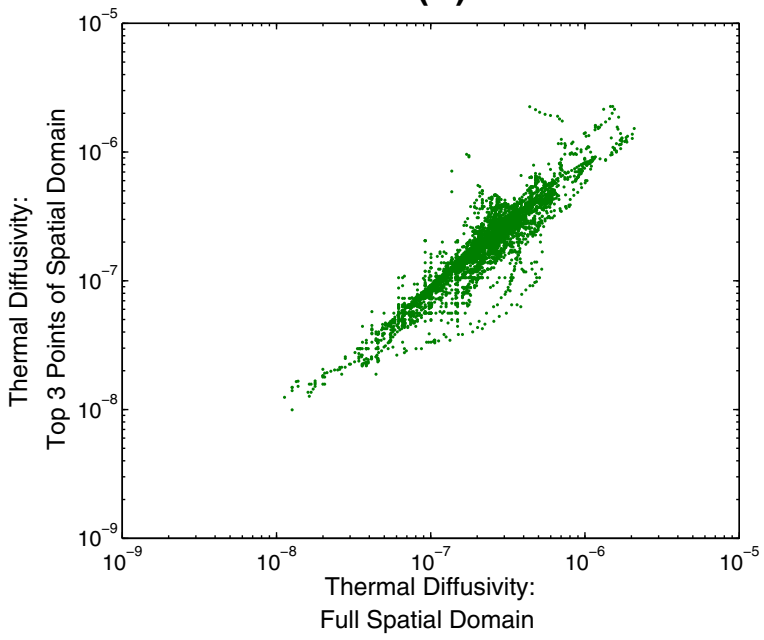

(d)

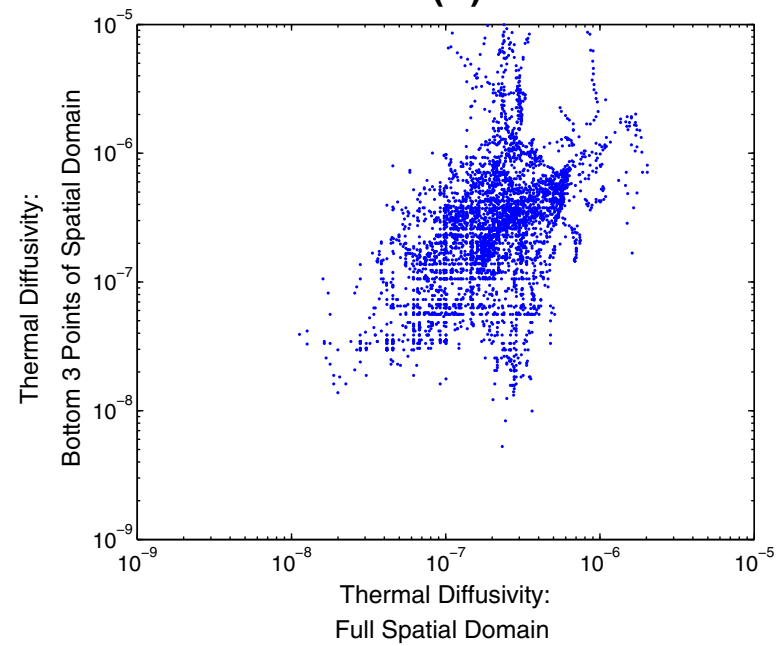

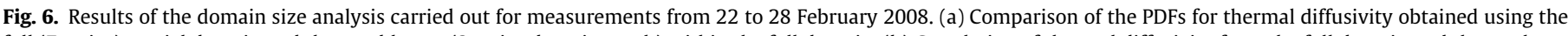

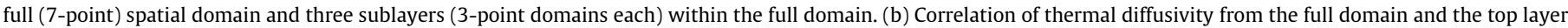
of the domain, (c) the middle layer of the domain and (d) the bottom layer of the domain.

resulting thermal diffusivities progressively decreased. However, the spikes and dips seen in Fig. 5 that approach $\sim 1 \times 10^{-6}$ and $\sim 1 \times 10^{-8}$ persisted. By reducing the small-scale temporal variability in these test cases, we were able to match the persistent extremes to times when the curvature in the temperature profiles switches concavity. Over a typical diurnal cycle, this occurs twice as the uppermost thermocouples, which show greater daily temperature change than those below it, switch from colder than those below to a warmer than those below, or visa versa (refer to Fig. 1 and inset of Fig. 4). This method appears incapable of solving for thermal diffusivities during these time periods, at least for temporal domains up to $12 \mathrm{~h}$.

Excluding the extreme values of thermal diffusivity produced when the temperature profiles switch concavity, the small scale temporal variations in thermal diffusivity could not be attributed (to the ability that the available information affords) to error in the temperature measurements, discretization error or to bulk layer approximations. This level of variability on $30 \mathrm{~min}$ time scales is not likely representative of time scales associated with changes of snow microstructure. The possibilities that this type of variability can be explained by non-diffusive forms of heat transfer such as wind pumping, convection, or radiation, remain open questions for future research. Since this method allows for high temporal resolution, it may prove useful in such studies, as well as studies regarding the expected temporal changes of thermal properties of snow.

A key outcome of these analyses is that the spatial resolution of the temperature measurements (or model discretization) has a greater effect on the resulting thermal diffusivities than noise in the temperature measurements. Hence, for future use of inversion-type techniques researchers should maximize the spatial resolution of the temperature measurements, as it will aid in adequately describing the curvature in the temperature profile and in capturing the peaks in the temperature profiles. In addition, high spatial resolution in the temperature measurements will provide more options for determining thermal diffusivity in various layers of the snowpack.

Use of this methodology to study the spatial variability of thermal diffusivity in large scale settings (for example, on the hydrologic catchment scale) is feasible. The proposed measurement technique, the TCT probe, was suitable, but not without flaws. For example, a snow temperature measurement technique which can guarantee no disruption to the surrounding snow, no additional heat conduction (through the poles or wires) and that the 


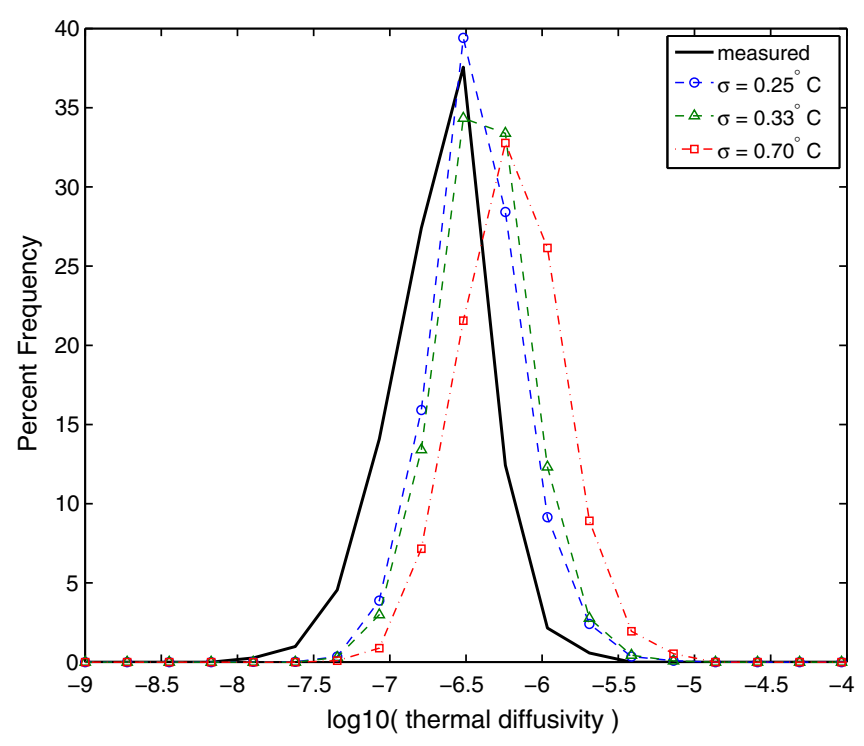

Fig. 7. Comparison of thermal diffusivity obtained from the temperature measurements for 22-28 February 2008, and these temperature measurements with added Gaussian noise, where $\sigma$ indicates the standard deviation of the added noise.

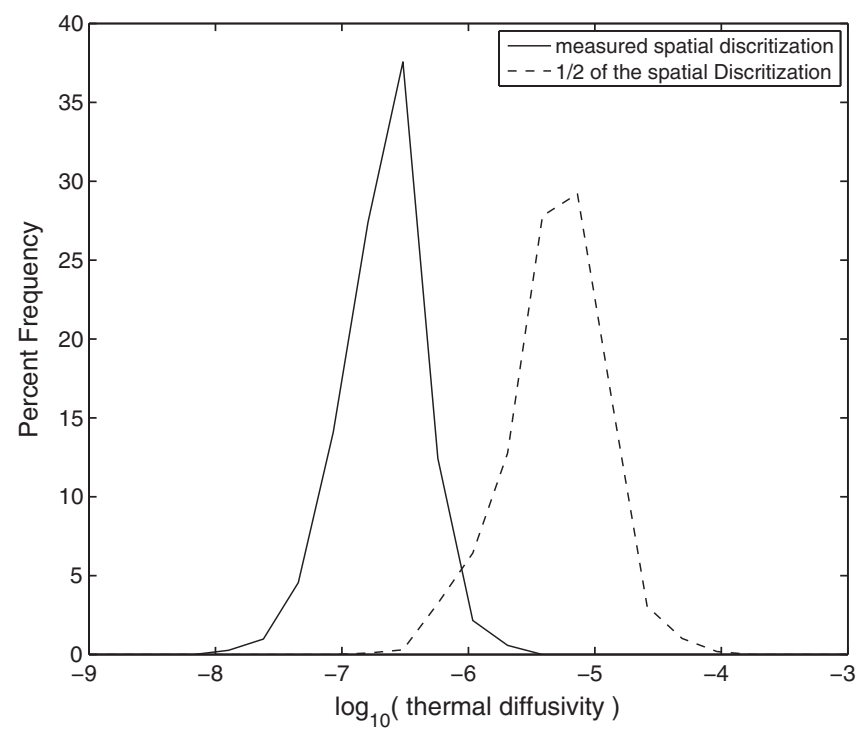

Fig. 8. Comparison of thermal diffusivity obtained with the spatial discretization of temperature measurements from 22 to 28 February 2008, and those obtained using half the spatial discretization (temperature measurement resolution) in solving the PDE.

measurements remain at a fixed location, could potentially improve this type of technique, as well as other studies where snow temperatures are measured. However, since our results compare well to widely-used, density-based parameterizations we believe these factors had little effect on our results.

\section{Conclusions}

Thermal diffusivity is an important thermophysical property that governs the heat transfer in snow. We have proposed a technique to obtain thermal diffusivity with high spatial and temporal resolution. The method uses temperature measurements for the initial condition and Neumann (heat flux) boundary conditions, and numerically solves the heat equation to iteratively optimize for thermal diffusivity. We found that using Neumann boundary conditions reduces the seemingly common problem of spurious, unrealistically high values of thermal diffusivity that arise when Dirichlet boundary conditions are used. Since the Neumann boundary conditions impose a flux instead of a temperature (as in Dirichlet boundary conditions), the modeled temperatures are not artificially constrained at the boundaries. This provided a significant improvement over the results using Dirichlet boundary conditions, in which thermal diffusivities were spread over 10 orders of magnitude. In addition, the new method imposes a statistical t-test ensuring the measured temperature profiles contain enough information such that the optimal thermal diffusivity is unique. An added consequence of including the t-test is that spuriously low values of thermal diffusivity are reduced.

The majority of the resulting thermal diffusivities compare well to published density-based empirical parameterizations. However, during time periods where the temperature profiles switch concavity this method behaves poorly and produces unreasonably high/ low results. With the exception of these spikes/dips, variability at 30 min time scales may indicate the presence of non-diffusive heat transfer processes such as wind pumping or convection. This method could assist in future studies of these phenomena. Using longer time intervals for the predicted temperatures naturally smooths out some of the variation in the results. However, heat transfer mechanisms and effects due to changes in the snow structure that occur on shorter time scales will be damped out.

An important result of this study is that high spatial resolution in the temperature measurements (leading to denser discretization in the model) appears to be the most significant parameter in using this method (and most likely other inversion methods) to estimate thermal diffusivity. Lower spatial resolution will tend to result in an erroneously higher thermal diffusivity to compensate for the reduced curvature in the temperature profiles. In general, researchers should be aware of this sensitivity for future uses of inversion techniques.

\section{Acknowledgements}

We are grateful to the anonymous reviewers and the associate editor, Michael Lehning, for their thoughtful comments that substantially improved the manuscript. This research was supported with partial funding from the Swiss National Science Foundation [200021_120238/1, 200020_125092/1], the NCCR Mobile Information Communication Systems (MICS) project and Competence Center Environment and Sustainability (CCES) of ETH Domain. We are also grateful for logistical support from the Crans-Montana ski resort, and for the field assistance from the many members of the Environmental Fluid Mechanics and Hydrology (EFLUM) laboratory at EPFL.

\section{References}

[1] Carlslaw HS, Jaeger JC. Conduction of heat in solids, vol. 2. Oxford, UK: Oxford University Press; 1959.

[2] Kondo J, Yamazaki T. A prediction model for snowmelt, snow surface temperature freezing depth using a heat balance method. J Appl Meteorol 1990;29(5):375-84.

[3] DeWalle DR, Rango A. Principles of snow hydrology. Cambridge University Press; 2008.

[4] Simoni S, Padoan S, Nadeau DF, Diebold M, Porporato A, Barrenetxea G, et al Hydrologic response of an alpine watershed: application of a meteorological wireless sensor network to understand streamflow generation. Water Resour Res 2011;47(W10524):W10524.

[5] Brandt RE, Warren SG. Solar-heating rates and temperature profiles in Antarctic snow and ice. J Glaciol 1993;39(131):99-110.

[6] Aoki T, Kuchiki K, Niwano M, Kodama Y, Hosaka M, Tanaka T. Physically based snow albedo model for calculating broadband albedos and the solar heating profile in snowpack for general circulation models. J Geophys Res 2011;116:D11114. 
[7] Sturm M, Johnson JB. Natural convection in the subarctic snow cover. J Geophys Res 1991;96(B7):11657-71.

[8] Zhekamukhov MK, Shukhova LZ. Convective instability of air in snow. J Appl Mech Tech Phys 1999;40(6):1042-7.

[9] Zhekamukhov MK, Zhekamukhova IM. On convective instability of air in the snow cover. J Eng Phys Thermophys 2002;75(4):849-58.

[10] Colbeck SC. Air movement in snow due to windpumping. J Glaciol 1989;35(120):209-13.

[11] Clarke GKC, Fisher DA, Waddington ED. Wind pumping: a potentially significant heat source in ice sheets, vol. 170, IAHS-AISH Publication, 1987.

[12] Calonne N, Flin F, Morin S, Lesaffre B, Rolland du Roscoat S, Geindreau C. Numerical and experimental investigations of the effective thermal conductivity of snow. Geophys Res Lett 2011;38(23):L23501.

[13] Arons EM, Colbeck SC. Geometry of heat and mass transfer in dry snow: a review of theory and experiment. Rev Geophys 1995;33(4):463-93.

[14] Riche F, Schneebeli M. Microstructural change around a needle probe to measure thermal conductivity of snow. J Glaciol 2010;56(199):871-6.

[15] Sturm M, Holmgren J, König M, Morris K. The thermal conductivity of seasonal snow. J Glaciol 1997:43(143):26-41.

[16] Brandt RE, Warren SG. Temperature measurements and heat transfer in nearsurface snow at the South Pole. J Glaciol 1997;43(144):339-51.

[17] Arons EM, Colbeck SC. Effective medium approximation for the conductivity of sensible heat in dry snow. Int J Heat Mass Trans. 1998;41(17):2653-66.

[18] Morris EM. Modelling the flow of mass and energy within a snowpack for hydrological forecasting. Ann Glaciol 1983;4:198-203.

[19] Pitman D, Zuckerman B. Effective Thermal Conductivity of Snow at $-88^{\circ} \mathrm{C}$,$27^{\circ} \mathrm{C}$ and $-5^{\circ} \mathrm{C}$. SAO Special, Report 267.

[20] Kaempfer TU, Schneebeli M, Sokratov SA. A microstructural approach to model heat transfer in snow. Geophys Res Lett 2005;32:21.

[21] Blackwell JH. A transient-flow method for determination of thermal constants of insulating materials in bulk Part I Theory. J Appl Phys 1954;25(2):137-44.

[22] Lange MA. Measurements of thermal parameters in Antarctic snow and firn. Ann Glaciol 1985;6:100-4.
[23] Morin S, Domine F, Arnaud L, Picard G. In-situ monitoring of the time evolution of the effective thermal conductivity of snow. Cold Reg. Sci. Tech. 2010;64:73-80

[24] Jaafar H, Picot JJC. Thermal conductivity of snow by a transient state probe method. Water Resour Res 1970;6(1):333-5.

[25] Sturm M, Johnson JB. Thermal conductivity measurements of depth hoar. J Geophys Res 1992;97(B2):2129-39.

[26] Aggarwal RK, Negi PS, Satyawali PK. New density-based thermal conductivity equation for snow. Defence Sci J 2009;59(2):126-30.

[27] Yen YC. Review of thermal properties of snow, ice and sea ice. Tech. Rep. 8110, CRRL, 1981.

[28] Rye CJ, Arnold NS, Willis IC, Kohler J. Modeling the surface mass balance of a high Arctic glacier using the ERA-40 reanalysis. J Geophys Res 2010;115(F2):F02014.

[29] Gilbert A, Wagnon P, Vincent C, Ginot P, Funk M. Atmospheric warming at a high-elevation tropical site revealed by englacial temperatures at Illimani, Bolivia $\left(6340 \mathrm{~m}\right.$ above sea level, $\left.16^{\circ} \mathrm{S}, 67^{\circ} \mathrm{W}\right)$. J Geophys Res 2010;115:D10109.

[30] Bakermans L, Jamieson B. SWarm: A simple regression model to estimate nearsurface snowpack warming for back-country avalanche forecasting. Cold Reg Sci Tech 2009;59(2-3):133-42.

[31] Fierz C, Lehning M. Assessment of the microstructure-based snow-cover model SNOWPACK: thermal and mechanical properties. Cold Reg Sci Tech 2001;33(2-3):123-31.

[32] Weller GE, Schwerdtfeger P. New data on the thermal conductivity of natural snow. J Glaciol 1971;10:309-11.

[33] Reusser DE, Zehe E. Low-cost monitoring of snow height and thermal properties with inexpensive temperature sensors. Hydrol Process 2011;25:1841-52

[34] Zhang T, Osterkamp TE. Considerations in determining thermal diffusivity from temperature time series using finite difference methods. Cold Reg Sci Tech 1995;23(4):333-41.

[35] <http://www.mathworks.com/help/techdoc/ref/pdepe.html>, 2011. 